Kırıkkale University, ozbek@kku.edu.tr, Kırıkkale-Turkey

http://dx.doi.org/10.12739/NWSA.2015.10.3.3C0132

\title{
OPERASYONEL REKABET DEĞERLENDİRMESİ (OCRA) YÖNTEMİYLE MEVDUAT BANKALARININ ETKINLİK ÖLÇÜMÜ
}

\section{ÖzET}

Finansal sistemin en temel kurumları olan bankaların etkin ve verimli çalışması hem bankacılık sektörünün karlılığı hem de ülke ekonomisi açısından büyük önem taşımaktadır. Bankalar, tasarrufların yatırıma dönüşürülmesinde aracılık eden finansal kurumlardır. Finans sektöründe hizmet veren bankalar arasında çok ciddi rekabet söz konusudur. Hizmet kalitesini sürekli iyileştirerek verimliliğini artıran bankalar bu yarışta her zaman bir adım önde olacaktır. Operational Competitiveness Rating (OCRA), benzer girdiler kullanarak benzer çıktılar üreten karar verme birimlerinin göreli etkinlik ölçümünde kullanılan oldukça kullanışlı ve yeni sayılabilecek bir yöntemdir. Bu çalışma ile Türkiye'de faaliyet gösteren 32 mevduat bankasının etkinliklerini ölçmek için 2011-2014 yıllarına ait "Bankalarımız Kitabından" alınan veriler kullanılarak OCRA yöntemine dayanan bir etkinlik ölçme model geliştirilmiştir. Modelde kullanılan girdi ve çıktı faktörleri literatür taraması sonucunda belirlenmiştir. Dört yılın tamamı dikkate alındığında en yüksek performansı Yapı ve Kredi Bankasının gösterdiği belirlenmiştir.

Anahtar Kelimeler: Operational Competitiveness Rating, OCRA, Etkinlik Ölçümü, Mevduat Bankaları

\section{EFFICIENCY MEASUREMENT OF DEPOSIT BANKS BY OPERATIONAL COMPETITIVENESS RATING (OCRA) METHOD}

\section{ABSTRACT}

The effective and efficient operation of deposit money banks, which are the most basic institutions of the financial system, is of great importance in terms of the profitability of the banking sector and the economy of the country. Banks are financial institutions that mediate the conversion of savings into investment. There is a very serious competition among banks serving in the financial sector. Those that increase their efficiency by continuously improving the service quality will be always one step ahead in this race. Competitiveness Operational Rating (OCRA) is a relatively new method that can be quite useful in measuring the relative efficiencies in decision making units by using similar input to produce similar output. This study aims to develop a model for efficiency measurement based on OCRA to measure the effectiveness of the 32 commercial banks operating in Turkey using data for the years 2011-2014 taken from the "Book for Banks". Input and output factors used in the model were determined according the results of a literature study. If you look at every four years, it has been established that YK showed the highest performance.

Keywords: Competitiveness Operational Rating, OCRA, Efficiency Measurement, Deposit Money Banks 


\section{GİRIŞ (INTRODUCTION)}

Tasarrufların değerlendirilmesinde ve aracılık işlemlerinin etkili olarak gerçekleştirilmesinde finansal sistemin en temel birimi olan bankacılık sektörünün etkin ve verimli çalışması ülke ekonomisi açısından büyük önem arz etmektedir. Ülke ekonomisinin merkezinde yer alan ve büyük öneme sahip olan bankacılık sisteminin rekabetten kaynaklanabilecek kar marjı azalmasına karşın etkinliğini sürekli olarak artırması gerekmektedir. İşletmeler açısından etkinlik ve verimlilik çalışmaları; maliyetleri düşürmek, rekabet avantajı elde etmek, karlılığı artırmak vb. nedenlerden dolayı büyük önem taşımaktadır. Özellikle finans sektöründe hizmet veren bankalar arasında günümüzde çok ciddi rekabet söz konusudur. Finansal sistemin vazgeçilmez kurumları olan bankaların verimliliği dikkate alınması gereken önemli bir konudur. Bu nedenle bu tür işletmelerin etkinliklerinin ölçülmesi gerekmektedir. Bankalar açısından etkinlik kavramı; temelde karlılığı, yeterli mevduat ve kredi düzeyini işaret etmekle birlikte, hedef düzeylerin sağlanabilmesinde ise banka için çok sayıda değişken ortaya çıkmaktadır. Banka bu değişkenleri bir arada yönetmek ve elde ettiği sonuçları rekabet koşulları çerçevesinde değerlendirmek zorundadır.

Bankaların göreli etkinliklerinin ölçülmesinde oran analizinin yanı sıra regresyon analizi gibi parametreli yöntemler ya da matematik programlama, Veri Zarflama Analizi (VZA), Operational Competitiveness Rating (OCRA), Çok Ölçütlü Karar Verme (ÇÖKV) gibi parametrik olmayan yöntemlerin farklı faktörler kullanılarak uygulandığı görülmektedir. Bankaların performansını ölçmeye yönelik olarak yapılan çalışmalarda ulusal muhasebe değerlerinin kullanılmadığı, üretim yaklaşımı ve aracılık yaklaşımı olmak üzere 2 temel yaklaşımla değişkenlerin seçildiği ifade edilmektedir (Colwell ve Davis, 1992:113; Berger ve Humphrey, 1997:197). Seçilen yaklaşıma göre bankaların etkinliklerini değerlendirmede farklı girdi ve çıktı faktörlerin belirlendikleri tespit edilmiştir.

Literatürde bankacılık sektörünün etkinliğini ölçmede pek çok çalışma yapıldığı görülmektedir. Güven ve Persentili (1997) Türkiye'deki bankaların performanslarını ölçmek için bankaların bilançolarını kullanarak doğrusal programlama temeline dayanan bir model geliştirmişlerdir. Spathis vd. (2002) ayrıştırma tekniğini önerirken, Ho (2006) Gri İlişkisel Analiz (Gİ) yöntemini; Garcia vd. (2010) ise Hedef Programlama (HP) tekniğini kullanmışlardır. Kaya (2001) Türkiye'deki bankaların etkinlik ölçümünde; Sangmi ve Nazir (2010) Hindistan'daki bankaların verimliliklerini analiz etmede; Ifeacho ve Ngalawa (2014) ise Güney Afrika'daki bankaların 1994-2011 yılları arasındaki performansını ölçmede CAMELS değerlendirme sistem yaklaşımını kullanmışlardır. Daly ve zhang(2014) Hon Kong'daki Çin sermayeli bankalar ile yerleşik ve yabancl sermayeli bankaların 20042010 yllları arasındaki etkinliklerini regresyon analizi yöntemi ile karşlaştırmıslardır. Chitan (2012) 2004-2011 yılları arasında Romanya'daki bankaların performansını ölçmek için çok değişkenli regresyon ve en küçük kareler yöntemini kullanmıştır.

Denizer vd. (2000), Işık vd. (2003), Demir ve Astarcıoğlu (2007), Erdem ve Erdem (2008) Türkiye'deki bankaların etkinliklerini; Thagunn ve Poudel (2013) Nepal bankalarının 2007-2011 yılları arasındaki verimliliklerini; Ayadi ve Ellouze (2013) ise Tunus bankalarının 1990-2009 yılları arasındaki performansını VZA ile ölçmüşlerdir. Ayrıca banka performansını ölçme ve değerlendirmede VZA tabanlı birçok çalışmaya literatürde rastlamak mümkündür. Bu çalışmalara örnek olarak Bauer vd. (1998); Camanho ve Dyson (2006); Portela ve Thanassoulis (2007); Pasiouras vd. (2008) tarafından 
Özbek, A.

NWSA-Social Sciences, 3C0132, 10, (3), 120-134.

yapılanlar sayılabilir. Diğer taraftan, Bauer vd. (1998) Amerikan bankalarının performansını VZA, SFA (Stochastic Frontier Analysis), TFA (Thick Frontier Approach) ve DFA (Distribution-Free Approach) yöntemlerini karşılaştırarak ölçmüşlerdir. Huang ve Wang (2002) Tayvan bankalarının etkinliklerini SFA, DFA ve VZA ile karşılaştırmalı olarak analiz etmişlerdir. Beccalli vd. (2006) SFA ve VZA; Parkan ve Wu (1999) VZA ile OCRA; Özbek (2015) Analtik Hiyerarşi Süreci (AHS) ve OCRA yöntemini banka etkinlik ölçümünde kullanmışlardır.

Tayvan bankalarının analizinde; Chen vd. (2013) bulanık VZA; Che vd. (2010) bulanık AHS ve VZA; Lin ve Chiu (2013)ise bağımsız bileşen analizi ve Network VZA modelini kullanmışlardır. Puhuong Ta ve Yin Har (2000) Singapur'daki bankaların, Stankevičiené ve Mencaitè (2012) ise Litwanya'daki bankaların performansını ölçmede AHS kullanmışlardır. Türkiye'deki bankaların etkinliklerini ölçmede; Bayyurt (2013) TOPSIS, ELECTRE III ve VZA kullanırken; Seçme vd. (2009), Akkoç ve Vatansever (2013) bulanık AHS kullanmışlardır. Bulanık AHS yöntemini, Ishizaka ve Nguyen (2013) ile Weifeng ve Huihuan (2008) da kullanmışlardır. Mahrooz vd. (2013) İran bankalarının performansını ölçmede bulanık AHS ve bulanık TOPSIS kullanırken, Mandic vd. (2014) Sırbıstan'daki bankacılık sektörünün performansını ölçmede bulanık AHS ve TOPSIS yöntemine dayanan bir model önermişlerdir. Mareschal ve Brans (1991); Mareschal ve Mertens (1992); Doumpos ve Zopounidis (2010) bankaların etkinliklerini ölçmede PROMETHEE yöntemini uygulamışlardır.

Bankacılık sektörünün etkinliğini ölçmede çok farklı yöntemlerin çok farklı faktörlerle kullanıldığı görülmesine rağmen Türkiye'deki mevduat bankaların etkinlik ölçümünde OCRA yönteminin kullanıldığı bir çalışmaya Özbek'in (2015) çalışması dışında başka bir çalışmaya literatürde rastlanmamıştır. Çalışmanın akışı şu şekildedir: ìkinci bölümde çalışmanın önemi değerlendirilmiştir. Üçüncü bölümde OCRA yönteminin teorik yapısı ele alınmış ve OCRA ile yapılan çalışmalara yer verilmiştir. Dördüncü bölümde ise Türkiye'de faaliyet gösteren mevduat bankalarının 2011-2014 yılı etkinlikleri, ilgili yıllara ait "Bankalarımı Kitabı" adlı kitaplardaki veriler temel alınarak ölçülmüştür. Son bölümde ise yapılan çalışma değerlendirilmiş ve bu konuda gelecekteki araştırmacılara öneriler sunulmuştur.

\section{2. ÇALIŞMANIN ÖNEMI (RESEARCH SIGNIFICANCE)}

Finansal sistemin etkin olarak çalışması kuruma fon sağlayanlar, fon talep edenler ve ülke ekonomisi açısından ciddiye alınması gereken bir konudur. Özellikle ülke ekonomisinde meydana gelecek krizlerde finans sektörünün etkinliği, krizi en az maliyetle atlatmakta belirleyici olmaktadır. Bu nedenle verimsiz olarak faaliyetlerini sürdüren bankaların önceden belirlenmesi ve performanslarını artırıcı tedbirler alınması göz önüne alınması gereken önemli bir konu olmaktadır. Bu açıdan yapılan bu çalışma, bankacılık sektöründe faaliyetlerini sürdüren finansal kurumların performanslarının tespit edilmesine katkı sağlamaktadır. Modelin uygulanması sonucu performansı düşük olan kurumlar, etkinliklerini artırmak için gerekli tedbirleri almalıdırlar.

Bankacılık sektörünün etkinliğini ölçmede çok farklı yöntemlerin ya tek başına ya da diğer yöntemlerle birlikte bütünleşik olarak çok farklı faktörler kullanılarak uygulandığı görülmüştür. Ancak Parkan ve Wu'nın (1999) ve Özbek'in (2015) yaptığı çalışmanın dışında OCRA yönteminin bankacılık sektöründe uygulandığı bir başka çalışmaya literatürde rastlanmamıştır. Türkiye'deki bankacılık sektöründe bu yöntemin tek başına uygulanması bir ilk olmaktadır. Bu açıdan bakıldığında literatüre katkı sağlamaktadır ve tarafımızdan önemli olarak değerlendirilmektedir. 
Özbek, A

\section{OPERASYONEL REKABET DEĞERLENDİRME YÖNTEMİ (OPERATIONAL COMPETITIVENESS RATING METHOD)}

OCRA, Parkan (1994) tarafından performans ölçümü, özellikle de verimlilik analizi problemlerinin çözümü için geliştirilmiş ve birçok alanda başarılı bir şekilde uygulanmış bir yöntemdir. Geliştirildiği tarihten bu tarafa birçok farklı karar birimlerine uygulanmıştır. Bu karar birimleri arasında; yazılım geliştirme ekibi (Parkan vd., 1997), endüstri işletmeleri(Parken ve Wu, 1999a), parfümeri marketleri (Parkan, 2003), oteller (Parkan, 2005), gida üretim tesisleri (Jayanthi vd., 1999), gibi kurumlar sayılabilir. OCRA, benzer karar birimlerinin bir dönem içerisinde verimliliğini ölçmenin yanı sıra tek bir karar biriminin birçok periyodu kapsayacak şekilde performans ölçümünde de kullanıldığı görülmüştür. Bu karar birimlerine kamu kurumları hizmet binaları (Parkan, 1999), yatırım bankacılığı (Parkan ve Wu, 1999b) ve toplu taşıma şirketleri (Parkan, 2002) örnek olarak verilebilir.

OCRA yönteminde, normalleştirme işleminde sıfıra bölme hatasını önlemek için girdi ve çıktı kriter değerlerinin pozitif olduğunu varsayar. $\boldsymbol{M}$ adet girdi ve $\boldsymbol{H}$ adet çıktı kriteri için önem ağırlıkları OCRA uygulayıcıları tarafından belirlenmelidir. Kriterlerin önem ağırlıklarını belirlemeye yönelik olarak OCRA tarafından bir yöntem gösterilmemiştir. Kriter önem ağırlıkları belirlenirken maliyet-fayda analizi gibi basit puanlama tekniği ya da AHS gibi ayrıntılı değerlendirme yöntemi kullanılabilir. Fayda-maliyet analizinde girdi ve çıktı kriterlerinin önem derecesini ölçek kullanarak belirlemek mümkündür. Örneğin $1^{\prime}$ den $5^{\prime}$ e kadar giden bir ölçekte, 5 en yüksek ağırlığı ifade ederken 1 en düşük ağırlığı ifade etmektedir.

Girdi ve çıktı kriter önem ağırlıklarını ifade eden $\boldsymbol{a}_{\boldsymbol{m}}$ ve $\boldsymbol{b}_{\boldsymbol{h}}$ değerlerinin toplamı 1 olmalıdır (Parkan, 2003). Bu, Eşitlik (1) ile gösterilmiştir. Kullanılan yönteme göre kriter önem ağırlıklarının toplamı 1 olmayabilir. Bu durumda her bir kriterin normalleştirilmemiş ağırlık değeri, toplam ağırlık değerine bölünerek normalleştirilmiş kriter önem ă̆ırlıkları elde edilir.

$$
\sum_{\mathrm{m}=1}^{\mathrm{M}} \mathrm{a}_{\mathrm{m}}+\sum_{\mathrm{h}=1}^{\mathrm{H}} \mathrm{b}_{\mathrm{h}}=1
$$

OCRA yönteminin işlem adımları 6 basamaktan oluşmaktadır ve bu adımlar aşağıda tanıtılmıştır (Parkan ve Wu, 2000; Peters ve Zelewski, 2010)

Adım 1: Ölçeklenmemiş girdi endeksin hesaplanması (Calculation of the unscaled input indices)

Girdi kriterleri dikkate alınarak her bir karar birimi için aşağıdaki (2) numaralı Eşitlik kullanılarak girdi endeksi hesaplanır. Bu amaçla her bir girdi kriteri $\boldsymbol{m}$ için karar birimi $\boldsymbol{k}^{\prime}$ nın girdi değeri olan $X_{m}^{k}$, karar birimlerinin en büyük girdi değerinden $\max _{n=1, \ldots K}\left(X_{m}^{n}\right)$ çıkarılır. Daha sonra bu çıkan sonuç karar birimlerinin en küçük girdi değerine $\min _{n=1, \ldots . K}\left(X_{m}^{n}\right)$ bölünerek normalleştirilir. Bu bölüm, girdi kriteri $\boldsymbol{m}^{\prime}$ nin ağırlığı olan $\boldsymbol{a}_{\boldsymbol{m}}$ ile çarpılır. Ortaya çıkan bu sonuç, ağırlıklı normalleştirilmiş girdi mesafe derecesi olarak adlandırılır. Mesafe dereceleri tüm girdiler ve karar birimleri için hesaplanır. Daha sonra ağırlıklı normalleştirilmiş girdi mesafe derecelerinin toplanmasıyla her bir karar birimi $\boldsymbol{k}$ için ölçeklenmemiş Girdi Endeksi $\boldsymbol{i}^{\boldsymbol{k}}$ hesaplanır.

$$
\mathrm{i}^{\mathrm{k}}=\sum_{\mathrm{m}=1}^{\mathrm{M}} \mathrm{a}_{\mathrm{m}} \frac{\max _{\mathrm{n}=1, \ldots, \mathrm{K}}\left(\mathrm{X}_{\mathrm{m}}^{\mathrm{n}}\right)-\mathrm{X}_{\mathrm{m}}^{\mathrm{k}}}{\min _{\mathrm{n}=1, \ldots, \mathrm{K}}\left(\mathrm{X}_{\mathrm{m}}^{\mathrm{n}}\right)}, \quad \forall \mathrm{n}=1, \ldots, \mathrm{K} ; \mathrm{X}_{\mathrm{m}}^{\mathrm{n}}>0 ; \forall \mathrm{k}=1, \ldots, \mathrm{K}
$$


Adım 2: Ölçeklenmemiş çıktı endeksin hesaplanması (Calculation of the unscaled output indices)

Çıktı kriterleri dikkate alınarak her bir karar birimi için aşağıdaki (3) numaralı Eşitlik kullanılarak çıktı endeksi hesaplanır. $\mathrm{Bu}$ amaçla her bir çıktı kriteri $\boldsymbol{h}$ için karar birimi $\boldsymbol{k}^{\prime}$ nın çıktı değeri olan $Y_{h}^{k}$ 'dan karar birimlerinin en küçük çıktı değeri olan $\min _{n=1}\left(Y_{h}^{n}\right)$ çıkarılır. Daha sonra bu çıkan sonuç karar birimlerinin en küçük çıktı değerine $\min _{n=1, \ldots, K}\left(Y_{h}^{n}\right)$ bölünerek normalleştirilir. Bu bölüm, çıktı kriteri $\boldsymbol{h}^{\prime} \mathrm{n} ı \mathrm{n}$ ağırlığı olan $\boldsymbol{b}_{\boldsymbol{h}}$ ile çarpılır. Ortaya çıkan bu sonuç, ağırlıklı normalleştirilmiş çıktı mesafe derecesi olarak adlandırılır. Mesafe dereceleri tüm çıktılar ve karar birimleri için hesaplanır. Daha sonra ağırlıklı normalleştirilmiş çıktı mesafe derecelerinin toplanmasıyla her bir karar birimi $\boldsymbol{k}$ için ölçeklenmemiş Çıktı Endeksi $\boldsymbol{o}^{\boldsymbol{k}}$ hesaplanır.

$$
\mathrm{o}^{\mathrm{k}}=\sum_{\mathrm{h}=1}^{\mathrm{H}} \mathrm{b}_{\mathrm{h}} \frac{\mathrm{Y}_{\mathrm{h}}^{\mathrm{k}}-\min _{\mathrm{n}=1, \ldots, \mathrm{K}} \mathrm{Y}_{\mathrm{h}}^{\mathrm{n}}}{\min _{\mathrm{n}=1, \ldots, \mathrm{K}} \mathrm{Y}_{\mathrm{h}}^{\mathrm{n}}}, \quad \forall \mathrm{n}=1, \ldots, \mathrm{K} ; \mathrm{Y}_{\mathrm{h}}^{\mathrm{n}}>0 ; \forall \mathrm{k}=1, \ldots, \mathrm{K}
$$

\section{Adım 3: Girdi endeksi ölçeklendirme (Scaling of the input indices)}

(4) numaralı Eşitlik kullanılarak bir karar biriminin $\boldsymbol{k}$. girdi endeks değerinden endeks setinin en küçük girdi endeks değeri çıkartılarak ölçeklendirme yapılır.

$$
\mathrm{I}^{\mathrm{k}}=\mathrm{i}^{\mathrm{k}}-\min _{\mathrm{n}=1, \ldots, \mathrm{K}} \mathrm{i}^{\mathrm{n}}, \quad \forall \mathrm{k}=1, \ldots, \mathrm{K}
$$

\section{Adım 4: Çıktı endeksi ölçeklendirme (Scaling of the output indices)}

(5) numaralı Eşitlik kullanılarak bir karar biriminin $\boldsymbol{k}$. çıktı endeks değerinden endeks setinin en küçük çıktı endeks değeri çıkartılarak ölçeklendirme yapılır.

$$
0^{\mathrm{k}}=\mathrm{o}^{\mathrm{k}}-\min _{\mathrm{n}=1, \ldots, \mathrm{K}} \mathrm{o}^{\mathrm{n}}, \quad \forall \mathrm{k}=1, \ldots, \mathrm{K}
$$

Adım 5: Ölçeklendirilmemiş etkinlik endeksin hesaplanması (Calculation of the unscaled efficiency indices)

(6) numaralı Eşitlik kullanılarak her bir karar birimi için ölçeklendirilmiş giriş endeks değeri ile çıktı endeks değeri toplanarak etkinlik endeksi hesaplanır.

$$
\mathrm{e}^{\mathrm{k}}=\mathrm{I}^{\mathrm{k}}+\mathrm{O}^{\mathrm{k}}, \quad \forall \mathrm{k}=1, \ldots, \mathrm{K}
$$

\section{Adım 6: Ölçeklendirilmiş etkinlik endeksin hesaplanması (Calculation of the scaled efficiency indices)}

(7) numaralı Eşitlik kullanılarak ölçeklendirilmemiş etkinlik endeksi elemanlarından en küçük eleman çıkartılarak ölçeklendirilmiş etkinlik endeksi hesaplanır.

$$
\mathrm{E}^{\mathrm{k}}=\mathrm{I}^{\mathrm{k}}+\mathrm{O}^{\mathrm{k}}-\min _{\mathrm{n}=1, \ldots, \mathrm{K}}\left(\mathrm{I}^{\mathrm{n}}+\mathrm{O}^{\mathrm{n}}\right), \quad \forall \mathrm{k}=1, \ldots, \mathrm{K}
$$

\section{BULGULAR VE TARTIŞMA (FINDINGS AND DISCUSSIONS)}

Bankaların 2011-2014 yıllarına ait etkinliğini ölçebilmek için Türkiye Bankalar Birliği'nin yayımladığı "Bankalarımız Kitabı" adlı kitaplardaki veriler kayak olarak alınmıştıri. Tablo 1 sadece 2014 yılına ait verileri göstermektedir. Literatür taraması neticesinde 4

\footnotetext{
1*: Kamu sermayeli bankalar **: Yerli sermayeli bankalar ***: Yabancl sermayeli bankalar
} 


\begin{abstract}
adet girdi ve 3 adet çıktı kriteri belirlenmiştir. Şube sayısı, Personel Sayısı, Mevduat, Faiz Giderleri girdi kriterlerini oluştururken; Kredi ve Alacaklar, Faiz Gelirleri ve Diğer Gelirler çıktı kriterlerini oluşturmuştur. Kriter ağırlıklarını belirlemede OCRA'da bir uygulama bulunmamaktadır. Girdi ve çıktı kriterlerinin ağırlıkları 1'den 5'e kadar giden bir ölçeğe göre belirlenmiştir. Ölçekte 5 en yüksek ağırlığı ifade ederken 1 en düşük ağırlığı ifade etmektedir. Kriter ağırlıkları aşağıda Tablo 2'de gösterilmiştir.
\end{abstract}

Tablo 1. 2014 yılına ait girdi ve çıktı verileri(.000)

( Table 1. Input and output data for year 2014(.000)

\begin{tabular}{|c|c|c|c|c|c|c|c|c|}
\hline \multicolumn{2}{|r|}{ Bankalar } & \multicolumn{4}{|c|}{ Girdi Faktörleri } & \multicolumn{3}{|c|}{ Çıktı Faktörleri } \\
\hline NR & Banka Adı & $\begin{array}{c}\text { Sube } \\
\text { Sayısı }\end{array}$ & $\begin{array}{c}\text { Personel } \\
\text { Sayısi }\end{array}$ & Mevduat & $\begin{array}{c}\text { Faiz } \\
\text { Giderleri }\end{array}$ & $\begin{array}{l}\text { Krediler ve } \\
\text { Alacaklar } \\
\end{array}$ & $\begin{array}{c}\text { Faiz } \\
\text { Gelirleri } \\
\end{array}$ & $\begin{array}{c}\text { Diğer } \\
\text { Gelirler }\end{array}$ \\
\hline 1 & Adabank $\star \star$ & 1 & 31 & 6.000 & 1 & 1 & 5.000 & \\
\hline 2 & Akbank ** & 990 & 16.305 & 113.373 .000 & 7.173 .000 & 125.978 .000 & 14.094 .000 & 2.994 .000 \\
\hline 3 & Alternatifbank $\star \star \star$ & 73 & 1.231 & 5.675 .000 & 590.000 & 7.882 .000 & 1.049 .000 & 122.000 \\
\hline 4 & Anadolubank $\star \star \star$ & 108 & 1.761 & 6.512 .000 & 438.000 & 6.249 .000 & 809.000 & 98.000 \\
\hline 5 & ${ }_{\star \star \star}^{\text {Arap-Türk Bankası }}$ & 7 & 282 & 3.108 .000 & 29.000 & 1.364 .000 & 138.000 & 56.000 \\
\hline 6 & 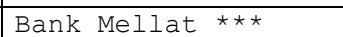 & 3 & 48 & 120.000 & 1 & 11.000 & 15.000 & 3.000 \\
\hline 7 & Bank of Tokya $* * \star$ & 1 & 58 & 1 & 1 & 1.197 .000 & 62.000 & 1 \\
\hline 8 & $\begin{array}{l}\text { Birleşik Fon } \\
\text { Bankası } \star * \star *\end{array}$ & 1 & 227 & 24.000 & 1 & 3.000 & 78.000 & 7.000 \\
\hline 9 & Burgan Bank $\star \star \star$ & 58 & 1.046 & 5.365 .000 & 402.000 & 6.448 .000 & 624.000 & 34.000 \\
\hline 10 & 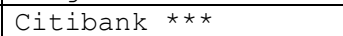 & 8 & 469 & 4.640 .000 & 182.000 & 2.414 .000 & 708.000 & 139.000 \\
\hline 11 & 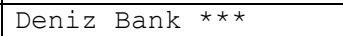 & 715 & 13.189 & 43.101 .000 & 2.838 .000 & 43.096 .000 & 5.744 .000 & 1.455 .000 \\
\hline 12 & 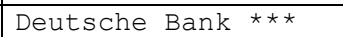 & 1 & 115 & 681.000 & 77.000 & 1.091 .000 & 224.000 & 58.000 \\
\hline 13 & Fibabank $\star \star$ & 67 & 1.222 & 5.246 .000 & 396.000 & 6.190 .000 & 708.000 & 29.000 \\
\hline 14 & Finans Bank $\star \star \star$ & 657 & 12.830 & 42.075 .000 & 3.408 .000 & 50.246 .000 & 6.496 .000 & 1.726 .000 \\
\hline 15 & 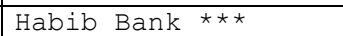 & 1 & 17 & 14.000 & 1 & 35.000 & 6.000 & 1.000 \\
\hline 16 & HSBC Bank $\star \star \star$ & 294 & 5.659 & 19.040 .000 & 1.360 .000 & 19.334 .000 & 2.590 .000 & 850.000 \\
\hline 17 & ING Bank $\star \star \star$ & 314 & 6.156 & 19.098 .000 & 1.461 .000 & 28.046 .000 & 3.166 .000 & 581.000 \\
\hline 18 & $\begin{array}{l}\text { JPMorgan Chase Bank } \\
\star \star \star \star\end{array}$ & 1 & 64 & 21.000 & 5.000 & 1 & 94.000 & 18.000 \\
\hline 19 & Odea Bank $\star \star \star$ & 48 & 1.388 & 21.061 .000 & 1.058 .000 & 18.011 .000 & 1.638 .000 & 46.000 \\
\hline 20 & $\begin{array}{l}\text { Société Générale } \\
\star \star \star\end{array}$ & 1 & 126 & 73.000 & 20.000 & 265.000 & 253.000 & 14.000 \\
\hline 21 & Sekerbank $\star \star$ & 312 & 4.460 & 13.539 .000 & 1.122 .000 & 14.633 .000 & 2.100 .000 & 431.000 \\
\hline 22 & Tekstil Bankası ** & 44 & 852 & 2.509 .000 & 161.000 & 2.799 .000 & 340.000 & 35.000 \\
\hline 23 & $\begin{array}{l}\text { The Royal Bank of } \\
\text { Scotland Plc } \star \star \star\end{array}$ & 1 & 76 & 514.000 & 26.000 & 381.000 & 217.000 & 95.000 \\
\hline 24 & Turkish Bank $\star \star ~$ & 18 & 265 & 906.000 & 50.000 & 896.000 & 94.000 & 7.000 \\
\hline 25 & Turkland Bank *** & 33 & 641 & 3.589 .000 & 260.000 & 3.365 .000 & 441.000 & 66.000 \\
\hline 26 & $\begin{array}{l}\text { Türk Ekonomi } \\
\text { Bankası } \star \star\end{array}$ & 546 & 10.142 & 39.439 .000 & 2.638 .000 & 45.392 .000 & 5.202 .000 & 1.111 .000 \\
\hline 27 & $\begin{array}{l}\text { Türkiye Cumhuriyeti } \\
\text { Ziraat Bankası * }\end{array}$ & 1.682 & 23.617 & 153.245 .000 & 9.558 .000 & 141.915 .000 & 18.165 .000 & 2.180 .000 \\
\hline 28 & $\begin{array}{l}\text { Türkiye Garanti } \\
\text { Bankası ** }\end{array}$ & 994 & 19.036 & 120.308 .000 & 7.643 .000 & 134.058 .000 & 15.086 .000 & 3.434 .000 \\
\hline 29 & $\begin{array}{l}\text { Türkiye Halk } \\
\text { Bankası * }\end{array}$ & 895 & 17.314 & 103.708 .000 & 6.340 .000 & 101.767 .000 & 11.451 .000 & 1.708 .000 \\
\hline 30 & $\begin{array}{l}\text { Türkiye İs Bankası } \\
\star \star\end{array}$ & 1.333 & 24.308 & 133.555 .000 & 8.632 .000 & 155.824 .000 & 16.086 .000 & 3.611 .000 \\
\hline 31 & $\begin{array}{l}\text { Türkiye Vakıflar } \\
\text { Bankası * }\end{array}$ & 890 & 14.920 & 91.757 .000 & 6.722 .000 & 104.584 .000 & 11.374 .000 & 2.121 .000 \\
\hline 32 & $\begin{array}{l}\text { Yapı ve Kredi } \\
\text { Bankası } \star \star\end{array}$ & 1.002 & 17.457 & 105.120 .000 & 6.164 .000 & 121.993 .000 .000 & 11.770 .000 & 2.972 .000 \\
\hline
\end{tabular}

Tablo 2. Kriter ağırlıkları

(Table 2. Weights of the criteria)

\begin{tabular}{|l|l|l|l|l|l|l|}
\hline $\begin{array}{l}\text { Sube } \\
\text { Sayısı }\end{array}$ & $\begin{array}{l}\text { Personel } \\
\text { Sayısı }\end{array}$ & Mevduat & $\begin{array}{l}\text { Faiz } \\
\text { Giderleri }\end{array}$ & $\begin{array}{l}\text { Kredi ve vacaklar } \\
\text { Alacala }\end{array}$ & $\begin{array}{l}\text { Faiz } \\
\text { Gelirleri }\end{array}$ & $\begin{array}{l}\text { Diğer } \\
\text { Gelirler }\end{array}$ \\
\hline 0,115 & 0,077 & 0,192 & 0,154 & 0,192 & 0,154 & 0,115 \\
\hline
\end{tabular}


Kriter ağırlıkları belirlendikten sonra ölçeklenmemiş girdi endeksleri hesaplanmıştır. Örneğin 10. karar birimi olan Citibank için endeks şu şekilde hesaplanmıştır:

$$
\begin{gathered}
i^{10}=0,115 * \frac{1.682-8}{1}+0,077 * \frac{24.308-469}{17}+0,192 * \frac{153.245 .000 .000-4.640 .000 .000}{1} \\
+0,154 * \frac{9.558 .000 .000-182.000 .000}{1}=29.976 .064 .361
\end{gathered}
$$

Tüm karar birimleri için girdi endeksi hesaplanmıştır. Aşağıda Tablo 3'de hesaplanan mesafe dereceleri ve girdi endeksleri gösterilmektedir.

\begin{tabular}{|c|c|c|c|c|c|c|}
\hline \multicolumn{2}{|r|}{ Bankalar } & \multicolumn{4}{|c|}{ Girdi Kriterleri } & \multirow[b]{2}{*}{ Girdi Endeksi } \\
\hline $\mathrm{Nr}$ & Banka Adı & $\begin{array}{c}\text { Şube } \\
\text { Sayısı }\end{array}$ & $\begin{array}{c}\text { Personel } \\
\text { Sayısı }\end{array}$ & Mevduat & $\begin{array}{c}\text { Faiz } \\
\text { Giderleri }\end{array}$ & \\
\hline 1 & Adabank ** & 194 & 110 & 29.421 .888 .000 & 1.471 .932 .000 & 30.893 .820 .304 \\
\hline 2 & Akbank ** & 80 & 36 & 7.655 .424 .000 & 367.290 .000 & 8.022 .714 .116 \\
\hline 3 & Alternatifbank $* \star \star *$ & 186 & 105 & 28.333 .440 .000 & 1.381 .072 .000 & 29.714 .512 .290 \\
\hline 4 & Anadolubank ** & 182 & 102 & 28.172 .736 .000 & 1.404 .480 .000 & 29.577 .216 .284 \\
\hline 5 & Arap-Türk Bankası *ᄎ* & 193 & 109 & 28.826 .304 .000 & 1.467 .466 .000 & 30.293 .770 .302 \\
\hline 6 & Bank Mellat *** & 194 & 110 & 29.400 .000 .000 & 1.471 .932 .000 & 30.871 .932 .303 \\
\hline 7 & Bank of Tokya *** & 194 & 110 & 29.423 .040 .000 & 1.471 .932 .000 & 30.894 .972 .303 \\
\hline 8 & Birleşik Fon Bankası $* \star \star \star *$ & 194 & 109 & 29.418 .432 .000 & 1.471 .932 .000 & 30.890 .364 .303 \\
\hline 9 & Burgan Bank $\star \star \star *$ & 187 & 105 & 28.392 .960 .000 & 1.410 .024 .000 & 29.802 .984 .293 \\
\hline 10 & 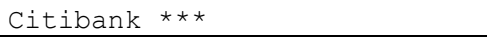 & 193 & 108 & 28.532 .160 .000 & 1.443 .904 .000 & 29.976 .064 .301 \\
\hline 11 & Deniz Bank $\star \star \star *$ & 112 & 50 & 21.147 .648 .000 & 1.034 .880 .000 & 22.182 .528 .162 \\
\hline 12 & Deutsche Bank $\star \star \star *$ & 194 & 110 & 29.292 .288 .000 & 1.460 .074 .000 & 30.752 .362 .304 \\
\hline 13 & Fibabank ** & 186 & 105 & 28.415 .808 .000 & 1.410 .948 .000 & 29.826 .756 .291 \\
\hline 14 & 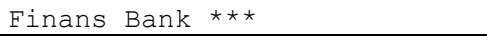 & 118 & 52 & 21.344 .640 .000 & 947.100 .000 & 22.291 .740 .170 \\
\hline 15 & 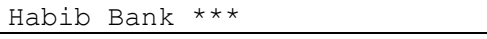 & 194 & 110 & 29.420 .352 .000 & 1.471 .932 .000 & 30.892 .284 .304 \\
\hline 16 & HSBC Bank $\star \star \star ~$ & 160 & 84 & 25.767 .360 .000 & 1.262 .492 .000 & 27.029 .852 .245 \\
\hline 17 & ING Bank $\star \star \star$ & 158 & 82 & 25.756 .224 .000 & 1.246 .938 .000 & 27.003 .162 .240 \\
\hline 18 & JPMorgan Chase Bank $\star \star \star *$ & 194 & 110 & 29.419 .008 .000 & 1.471 .162 .000 & 30.890 .170 .304 \\
\hline 19 & Odea Bank *** & 189 & 104 & 25.379 .328 .000 & 1.309 .000 .000 & 26.688 .328 .292 \\
\hline 20 & Société Générale $\star * \star$ & 194 & 110 & 29.409 .024 .000 & 1.468 .852 .000 & 30.877 .876 .304 \\
\hline 21 & Şekerbank ** & 158 & 90 & 26.823 .552 .000 & 1.299 .144 .000 & 28.122 .696 .248 \\
\hline 22 & Tekstil Bankası ** & 189 & 106 & 28.941 .312 .000 & 1.447 .138 .000 & 30.388 .450 .295 \\
\hline 23 & The Royal B. of Scotland $* \star *$ & 194 & 110 & 29.324 .352 .000 & 1.467 .928 .000 & 30.792 .280 .304 \\
\hline 24 & Turkish Bank ** & 192 & 109 & 29.249 .088 .000 & 1.464 .232 .000 & 30.713 .320 .301 \\
\hline 25 & Turkland Bank *** & 190 & 107 & 28.733 .952 .000 & 1.431 .892 .000 & 30.165 .844 .297 \\
\hline 26 & Türk Ekonomi Bankası ** & 131 & 64 & 21.850 .752 .000 & 1.065 .680 .000 & 22.916 .432 .195 \\
\hline 27 & T.C. Ziraat Bankası* & 0 & 3 & 0 & 0 & 3 \\
\hline 28 & Türkiye Garanti Bankası ** & 79 & 24 & 6.323 .904 .000 & 294.910 .000 & 6.618 .814 .103 \\
\hline 29 & Türkiye Halk Bankası * & 91 & 32 & 9.511 .104 .000 & 495.572 .000 & 10.006 .676 .122 \\
\hline 30 & Türkiye İş Bankası ** & 40 & 0 & 3.780 .480 .000 & 142.604 .000 & 3.923 .084 .040 \\
\hline 31 & Türkiye Vakıflar Bankası * & 91 & 43 & 11.805 .696 .000 & 436.744 .000 & 12.242 .440 .134 \\
\hline 32 & Yapı ve Kredi Bankası ** & 78 & 31 & 9.240 .000 .000 & 522.676 .000 & 9.762 .676 .110 \\
\hline
\end{tabular}

Tablo 3. Ölçeklenmemiş girdi endeksi

(Table 3. Unscaled input indizes)

Benzer şekilde ölçeklenmemiş çıktı endeksleri hesaplanmıştır. Örneğin 10. karar birimi olan Citibank için çıktı endeksi şu şekilde hesaplanmıştır:

$$
o^{10}=0,192 * \frac{2.414 .000 .000-1}{1}+0,154 * \frac{708.000 .000-5.000 .000}{5.000 .000}+0,115 * \frac{139.000 .000-1}{1}
$$


Özbek, A

NWSA-Social Sciences, 3C0132, 10, (3), 120-134.

Tüm karar birimleri için hesaplanan ölçeklenmemiş çıktı endeksi Tablo 4'de gösterilmiştir.

Bir sonraki adımda (4) numaralı Eşitlik kullanılarak ölçeklendirme yapılmıştır. Ölçeklendirme sayesinde karar birimleri arasında en düşük değere sahip olanın 0 değeri alması sağlanır. Örneğin Citibank için hesaplama şu şekilde yapılmıştır. Citibank'ın girdi endeks değerinden, T.C. Ziraat Bankasına ait olan en düşük endeks değeri çıkartılmıştır.

$$
I^{10}=29.976 .064 .301-3=29.976 .064 .298
$$

Benzer şekilde karar birimlerinin çıktı endeks değerleri içinde ölçeklendirme yapılmıştır. Ancak karar birimlerinden olan Adabank zaten en düşük değer olarak 0 içerdiğinden, bu durumda çıtı endekslerini ölçeklendirmeye gerek kalmamıştır. Tablo 5' ölçeklendirilmiş girdi, Tablo 6 ise çıktı endeksini göstermektedir.

Tablo 4. Ölçeklenmemiş çıktı endeksi

(Table 4. unscaled output indizes)

\begin{tabular}{|c|c|c|c|c|c|}
\hline \multicolumn{2}{|r|}{ Bankalar } & \multicolumn{3}{|c|}{ Çıktı Kriterleri } & \multirow[b]{2}{*}{ Çıktı Endeksi } \\
\hline $\mathrm{Nr}$ & Banka Adı & \begin{tabular}{|l|} 
Krediler ve \\
Alacaklar \\
\end{tabular} & $\begin{array}{c}\text { Faiz } \\
\text { Gelirleri } \\
\end{array}$ & Diğer Gelirler & \\
\hline 1 & Adabank ** & - & - & - & - \\
\hline 2 & Akbank ** & 24.187 .776 .000 & 434 & 344.310 .000 & 24.532 .086 .434 \\
\hline 3 & Alternatifbank *** & 1.513 .344 .000 & 32 & 14.030 .000 & 1.527 .374 .032 \\
\hline 4 & Anadolubank ** & 1.199 .808 .000 & 25 & 11.270 .000 & 1.211 .078 .024 \\
\hline 5 & Arap-Türk Bankası *** & 261.888 .000 & 4 & 6.440 .000 & 268.328 .004 \\
\hline 6 & 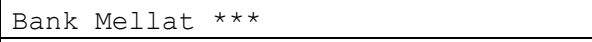 & 2.112 .000 & 0 & 345.000 & 2.457 .000 \\
\hline 7 & Bank of Tokya *** & 229.824 .000 & 2 & 0 & 229.824 .002 \\
\hline 8 & 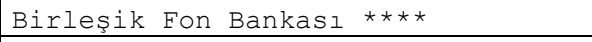 & 576.000 & 2 & 805.000 & 1.381 .002 \\
\hline 9 & Burgan Bank *** & 1.238 .016 .000 & 19 & 3.910 .000 & 1.241 .926 .019 \\
\hline 10 & Citibank *** & 463.488 .000 & 22 & 15.985 .000 & 479.473 .021 \\
\hline 11 & Deniz Bank *** & 8.274 .432 .000 & 177 & 167.325 .000 & 8.441 .757 .176 \\
\hline 12 & Deutsche Bank $* \star \star$ & 209.472 .000 & 7 & 6.670 .000 & 216.142 .006 \\
\hline 13 & Fibabank $\star \star$ & 1.188 .480 .000 & 22 & 3.335 .000 & 1.191 .815 .021 \\
\hline 14 & Finans Bank $\star \star \star$ & 9.647 .232 .000 & 200 & 198.490 .000 & 9.845 .722 .200 \\
\hline 15 & Habib Bank *** & 6.720 .000 & 0 & 115.000 & 6.835 .000 \\
\hline 16 & HSBC Bank *** & 3.712 .128 .000 & 80 & 97.750 .000 & 3.809 .878 .079 \\
\hline 17 & ING Bank *** & 5.384 .832 .000 & 97 & 66.815 .000 & 5.451 .647 .097 \\
\hline 18 & JPMorgan Chase Bank *** & 0 & 3 & 2.070 .000 & 2.070 .003 \\
\hline 19 & Odea Bank *** & 3.458 .112 .000 & 50 & 5.290 .000 & 3.463 .402 .050 \\
\hline 20 & 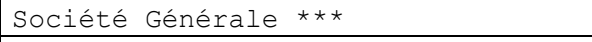 & 50.880 .000 & 8 & 1.610 .000 & 52.490 .007 \\
\hline 21 & Şekerbank ** & 2.809 .536 .000 & 65 & 49.565 .000 & 2.859 .101 .064 \\
\hline 22 & Tekstil Bankası ** & 537.408 .000 & 10 & 4.025 .000 & 541.433 .010 \\
\hline 23 & The Royal Bank of Scotland Plc *** & 73.152 .000 & 7 & 10.925 .000 & 84.077 .006 \\
\hline 24 & Turkish Bank ** & 172.032 .000 & 3 & 805.000 & 172.837 .002 \\
\hline 25 & Turkland Bank *** & 646.080 .000 & 13 & 7.590 .000 & 653.670 .013 \\
\hline 26 & Türk Ekonomi Bankası ** & 8.715 .264 .000 & 160 & 127.765 .000 & 8.843 .029 .160 \\
\hline 27 & T.C. Ziraat Bankası * & 27.247 .680 .000 & 559 & 250.700 .000 & 27.498 .380 .559 \\
\hline 28 & Türkiye Garanti Bankası ** & 25.739 .136 .000 & 464 & 394.910 .000 & 26.134 .046 .464 \\
\hline 29 & Türkiye Halk Bankası * & 19.539 .264 .000 & 353 & 196.420 .000 & 19.735 .684 .352 \\
\hline 30 & Türkiye İş Bankası ** & 29.918 .208 .000 & 495 & 415.265 .000 & 30.333 .473 .495 \\
\hline 31 & Türkiye Vakıflar Bankası * & 20.080 .128 .000 & 350 & 243.915 .000 & 20.324 .043 .350 \\
\hline 32 & Yapı ve Kredi Bankası ** & 23.422 .656 .000 & 362 & 341.780 .000 & 23.764 .436 .362 \\
\hline
\end{tabular}


Tablo 5. Ölçeklendirilmiş girdi endeksi (Table 5. Scaled input indices)

\begin{tabular}{|c|c|c|c|c|c|}
\hline $\mathrm{Nr}$ & Banka Adı & Girdi Endeksi & $\mathrm{Nr}$ & Banka Adı & Girdi Endeksi \\
\hline 1 & Adabank ** & 30.893 .820 .301 & 17 & ING Bank $\star \star \star *$ & 27.003 .162 .237 \\
\hline 2 & Akbank ** & 8.022 .714 .113 & 18 & JPMorgan Chase Bank *** & 30.890 .170 .301 \\
\hline 4 & Anadolubank ** & 29.577 .216 .281 & 20 & Société Générale *** & 30.877 .876 .300 \\
\hline 5 & Arap-Türk Bankası *** & 30.293 .770 .299 & 21 & Şekerbank ** & 28.122 .696 .245 \\
\hline 8 & $\begin{array}{l}\text { Birleşik Fon Bankası } \\
\star \star \star \star\end{array}$ & 30.890 .364 .300 & 24 & Turkish Bank ** & 30.713 .320 .298 \\
\hline 9 & Burgan Bank *** & 29.802 .984 .290 & 25 & Turkland Bank *** & 30.165 .844 .294 \\
\hline 10 & Citibank *** & 29.976 .064 .298 & 26 & Türk Ekonomi Bankası ** & 22.916 .432 .192 \\
\hline 14 & Finans Bank $* * *$ & 22.291 .740 .167 & 30 & Türkiye İş Bankası ** & 3.923 .084 .037 \\
\hline 15 & Habib Bank *** & 30.892 .284 .301 & 31 & Türkiye Vafıflar Bankası * & 12.242 .440 .131 \\
\hline 16 & HSBC Bank *** & 27.029 .852 .242 & 32 & Yapı ve Kredi Bankası ** & 9.762 .676 .106 \\
\hline
\end{tabular}

Girdi ve çıktı için ölçeklendirilmiş endeks oluşturulduktan sonra Eşitlik (6)kullanllarak ölçeklendirilmemiş etkinlik endeksi oluşturulur. Örneğin Citibank için ölçeklendirilmemiş etkinlik endeksi şu şekilde oluşturulmuştur:

$$
E^{10}=29.976 .064 .298+479.473 .021=30.455 .537 .319
$$

Karar birimlerinin ölçeklendirilmemiş etkinlik endeksi Tablo 7'de gösterilmiştir.

Son olarak (7) numaralı Eşitlik kullanılarak ölçeklendirilmemiş etkinlik endeksi elemanlarından en küçük eleman çıkartılarak ölçeklendirilmiş etkinlik endeksi hesaplanmıştır.

Karar birimlerinin ölçeklendirilmiş etkinlik endeksi Tablo 8'de gösterilmiştir.

Karar birimleri, etkinlik endeksine göre büyükten küçüğe doğru sıralanır. En yüksek değeri alan karar birimi en iyi performansı gösteriyor demektir. Tablo 9 karar birimlerinin yıllara göre performans sıralamasını göstermektedir. OCRA temelli modelin bankacılık sektörüne uygulanması sonucunda en etkin bankanın Yapı ve Kredi Bankası olduğu belirlenmiştir. En verimsiz bankanın ise Türkiye Cumhuriyeti Ziraat Bankası olduğu görülmüştür. 
Tablo 6. Ölçeklendirilmiş çıktı endeksi (Table 6. Scaled output indices)

\begin{tabular}{|c|c|c|c|c|c|}
\hline $\mathrm{Nr}$ & Banka Adı & Çıktı Endeksi & $\mathrm{Nr}$ & Banka Adı & Çıktı Endeksi \\
\hline 1 & Adabank ** & 0 & 17 & ING Bank $\star \star \star *$ & 5.451 .647 .097 \\
\hline 2 & Akbank ** & 24.532 .086 .434 & 18 & JPMorgan Chase Bank *** & 2.070 .003 \\
\hline 3 & Alternatifbank $* * *$ & 1.527 .374 .032 & 19 & Odea Bank $\star \star \star$ & 3.463 .402 .050 \\
\hline 4 & Anadolubank ** & 1.211 .078 .024 & 20 & Société Générale *** & 52.490 .007 \\
\hline 5 & Arap-Türk Bankası $* * \star$ & 268.328 .004 & 21 & Şekerbank ** & 2.859 .101 .064 \\
\hline 6 & Bank Mellat $\star \star \star *$ & 2.457 .000 & 22 & Tekstil Bankası ** & 541.433 .010 \\
\hline 7 & Bank of Tokya *** & 229.824 .002 & 23 & $\begin{array}{l}\text { The Royal Bank of Scotland } \\
\text { Plc } \star \star \star\end{array}$ & 84.077 .006 \\
\hline 8 & $\begin{array}{l}\text { Birleşik Fon Bankası } \\
\star \star \star \star\end{array}$ & 1.381 .002 & 24 & Turkish Bank ** & 172.837 .002 \\
\hline 9 & Burgan Bank *** & 1.241 .926 .019 & 25 & 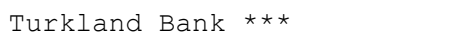 & 653.670 .013 \\
\hline 10 & Citibank $\star * \star$ & 479.473 .021 & 26 & Türk Ekonomi Bankası ** & 8.843 .029 .160 \\
\hline 11 & Deniz Bank *** & 8.441 .757 .176 & 27 & T.C. Ziraat Bankası * & 27.498 .380 .559 \\
\hline 12 & Deutsche Bank $* \star *$ & 216.142 .006 & 28 & Türkiye Garanti Bankası ** & 26.134 .046 .464 \\
\hline 13 & Fibabank ** & 1.191 .815 .021 & 29 & Türkiye Halk Bankası * & 19.735 .684 .352 \\
\hline 14 & Finans Bank $\star \star \star$ & 9.845 .722 .200 & 30 & Türkiye İş Bankası ** & 30.333 .473 .495 \\
\hline 15 & Habib Bank *** & 6.835 .000 & 31 & Türkiye Vafıflar Bankası * & 20.324 .043 .350 \\
\hline 16 & HSBC Bank *** & 3.809 .878 .079 & 32 & Yapı ve Kredi Bankası ** & 23.764 .436 .362 \\
\hline
\end{tabular}

Tablo 7. Ölçeklendirilmemiş etkinlik endeksi

(Table 7. Unscaled performance indices)

\begin{tabular}{|c|c|c|c|c|c|}
\hline $\mathrm{Nr}$ & Banka Adı & $\begin{array}{l}\text { Etkinlik } \\
\text { Endeksi }\end{array}$ & $\mathrm{Nr}$ & Banka Adı & $\begin{array}{l}\text { Etkinlik } \\
\text { Endeksi }\end{array}$ \\
\hline 1 & Adabank ** & 30.893 .820 .301 & 17 & ING Bank *** & 32.454 .809 .334 \\
\hline 2 & Akbank ** & 32.554 .800 .547 & 18 & JPMorgan Chase Bank *** & 30.892 .240 .303 \\
\hline 3 & Alternatifbank $* * *$ & 31.241 .886 .319 & 19 & Odea Bank *** & 30.151 .730 .339 \\
\hline 4 & Anadolubank ** & 30.788 .294 .305 & 20 & Société Générale *** & 30.930 .366 .308 \\
\hline 5 & Arap-Türk Bankası *** & 30.562 .098 .303 & 21 & Şekerbank ** & 30.981 .797 .309 \\
\hline 6 & Bank Mellat $* \star *$ & 30.874 .389 .300 & 22 & Tekstil Bankası ** & 30.929 .883 .302 \\
\hline 7 & Bank of Tokya $\star \star \star *$ & 31.124 .796 .302 & 23 & $\begin{array}{l}\text { The Royal Bank of Scotland } \\
\text { Plc *** }\end{array}$ & 30.876 .357 .307 \\
\hline 8 & $\begin{array}{l}\text { Birleşik Fon Bankası } \\
\star \star \star \star\end{array}$ & 30.891 .745 .302 & 24 & Turkish Bank ** & 30.886 .157 .300 \\
\hline 9 & Burgan Bank $\star \star \star *$ & 31.044 .910 .308 & 25 & Turkland Bank *** & 30.819 .514 .307 \\
\hline 10 & Citibank $\star \star \star *$ & 30.455 .537 .319 & 26 & Türk Ekonomi Bankası ** & 31.759 .461 .352 \\
\hline 11 & Deniz Bank *** & 30.624 .285 .335 & 27 & T.C. Ziraat Bankası * & 27.498 .380 .559 \\
\hline 12 & Deutsche Bank $\star \star \star *$ & 30.968 .504 .307 & 28 & Türkiye Garanti Bankası ** & 32.752 .860 .564 \\
\hline 13 & Fibabank ** & 31.018 .571 .309 & 29 & Türkiye Halk Bankası * & 29.742 .360 .472 \\
\hline 14 & Finans Bank $* \star *$ & 32.137 .462 .367 & 30 & Türkiye İş Bankası ** & 34.256 .557 .532 \\
\hline 15 & Habib Bank *** & 30.899 .119 .300 & 31 & Türkiye Vafıflar Bankası * & 32.566 .483 .481 \\
\hline 16 & HSBC Bank *** & 30.839 .730 .321 & 32 & Yapı ve Kredi Bankası ** & 33.527 .112 .468 \\
\hline
\end{tabular}


Tablo 8. Ölçeklendirilmiş etkinlik endeksi

(Table 8. Scaled performance indices)

\begin{tabular}{|c|c|c|c|c|c|}
\hline $\mathrm{Nr}$ & Banka Adı & Etkinlik Endeksi & $\mathrm{Nr}$ & Banka Adı & Etkinlik Endeksi \\
\hline 1 & Adabank ** & 3.395 .439 .742 & 17 & ING Bank $* * *$ & 4.956 .428 .775 \\
\hline 2 & Akbank ** & 5.056 .419 .988 & 18 & JPMorgan Chase Bank *** & 3.393 .859 .744 \\
\hline 3 & Alternatifbank $* \star \star$ & 3.743 .505 .760 & 19 & Odea Bank $\star \star \star$ & 2.653 .349 .780 \\
\hline 4 & Anadolubank ** & 3.289 .913 .746 & 20 & Société Générale *** & 3.431 .985 .749 \\
\hline 5 & Arap-Türk Bankası *** & 3.063 .717 .744 & 21 & Şekerbank ** & 3.483 .416 .750 \\
\hline 6 & 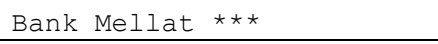 & 3.376 .008 .741 & 22 & Tekstil Bankası ** & 3.431 .502 .743 \\
\hline 7 & Bank of Tokya *** & 3.626 .415 .743 & 23 & The Royal Bank of Scotland Plc *** & 3.377 .976 .748 \\
\hline 8 & 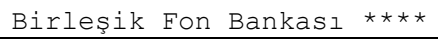 & 3.393 .364 .743 & 24 & Turkish Bank ** & 3.387 .776 .741 \\
\hline 9 & 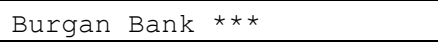 & 3.546 .529 .749 & 25 & Turkland Bank $\star \star \star$ & 3.321 .133 .748 \\
\hline 10 & Citibank $* \star \star$ & 2.957 .156 .760 & 26 & Türk Ekonomi Bankası ** & 4.261 .080 .793 \\
\hline 11 & Deniz Bank $* \star \star$ & 3.125 .904 .776 & 27 & T.C. Ziraat Bankası * & 0 \\
\hline 12 & Deutsche Bank $\star \star \star *$ & 3.470 .123 .748 & 28 & Türkiye Garanti Bankası ** & 5.254 .480 .005 \\
\hline 13 & Fibabank ** & 3.520 .190 .750 & 29 & Türkiye Halk Bankası * & 2.243 .979 .913 \\
\hline 14 & Finans Bank $* \star \star$ & 4.639 .081 .808 & 30 & Türkiye İş Bankası ** & 6.758 .176 .973 \\
\hline 15 & Habib Bank *** & 3.400 .738 .741 & 31 & Türkiye Vakıflar Bankası * & 5.068 .102 .922 \\
\hline 16 & HSBC Bank *** & 3.341 .349 .762 & 32 & Yapı ve Kredi Bankası ** & 6.028 .731 .909 \\
\hline
\end{tabular}

Tablo 9. Yıllara göre etkinlik endeksi

(Table 9. Performance indices by years)

\begin{tabular}{|c|c|c|c|c|c|c|c|c|}
\hline Ylllar & 2011 & & 2012 & & 2013 & & 2014 & \\
\hline Bankalar & Değerler & 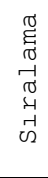 & Değerler & 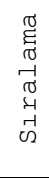 & Değerler & 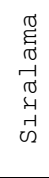 & Değerler & \\
\hline Adabank ** & 7.068 .014 .039 & 18 & $7.917 .460 .952,3$ & 20 & 6.644 .205 .748 & 20 & 3.395 .439 .742 & 18 \\
\hline Akbank $\star \star ~$ & .688 .698 .754 & 26 & $9.270 .353 .587,6$ & 5 & 7.168 .634 .000 & 6 & .056 .419 .988 & 5 \\
\hline Alternatifbank $* \star \star *$ & 7.245 .154 .944 & 8 & $8.190 .130 .852,7$ & 11 & 6.874 .391 .763 & 10 & 3.743 .505 .760 & 9 \\
\hline Anadolubank $\star \star *$ & 7.135 .386 .885 & 11 & $8.037 .417 .788,4$ & 14 & 6.631 .140 .749 & 22 & 3.289 .913 .746 & 26 \\
\hline 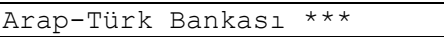 & .879 .023 .022 & 23 & $7.880 .314 .933,0$ & 27 & 9.178 .749 & 28 & 3.063 .717 .744 & 28 \\
\hline Bank Mellat $\star \star \star$ & .599 .781 .038 & 28 & $7.889 .744 .950,0$ & 24 & 6.623 .468 .748 & 24 & 6.008 .741 & 23 \\
\hline Bank of Tokya $* \star \star$ & 7.068 .742 .042 & 0 & $7.917 .842 .954,6$ & 0 & 6.647 .853 .748 & 19 & 3.626 .415 .743 & 10 \\
\hline 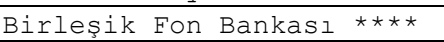 & 7.074 .700 .023 & 16 & $7.925 .155 .937,1$ & 18 & 6.646 .695 .748 & 18 & 3.393 .364 .743 & 20 \\
\hline Burgan Bank $\star \star \star$ & 7.103 .217 .962 & 12 & $7.881 .730 .872,7$ & 26 & 6.832 .604 .752 & 12 & 3.546 .529 .749 & 11 \\
\hline 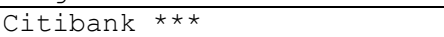 & 6.615 .540 .866 & 27 & $7.598 .906 .787,0$ & 29 & 6.223 .544 .762 & 30 & 2.957 .156 .760 & 29 \\
\hline 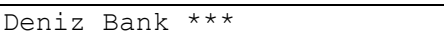 & 87.222 & 4 &, 9 & 8 & 7.03 & 9 & 904.776 & 27 \\
\hline Deutsche Bank $* \star *$ & .613 .034 & 10 & 46,5 & 16 & 6.70 & 15 & 123.748 & 14 \\
\hline Fibabank $\star \star$ & 7.074 .719 .006 & 15 & $8.041 .694 .904,4$ & 13 & 6.728 .228 .753 & 14 & 0.190 .750 & 12 \\
\hline Finans Bank $\star \star \star$ & 7.715 .936 .148 & 6 & $9.197 .700 .959,1$ & 6 & 7.261 .438 .825 & 5 & 4.639 .081 .808 & 7 \\
\hline 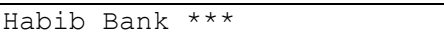 & 7.072 .929 .041 & 17 & $7.922 .759 .953,3$ & 19 & 6.651 .462 .748 & 17 & 3.400 .738 .741 & 17 \\
\hline HSBC Bank $\star \star \star$ & 531 & 7 & 1 & 10 & 6.85 & 11 & 349.762 & 24 \\
\hline ING Bank $* \star \star$ & 38.603 & 2 & 08,9 & 7 & .336 .776 & 4 & .428 .775 & 6 \\
\hline 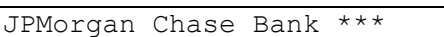 & .773 .850 .038 & 25 & $7.910 .781 .950,5$ & 23 & .664 .749 & 21 & .859 .744 & 29 \\
\hline 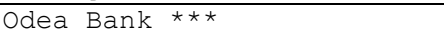 & 7.068 .742 .042 & 0 & $7.772 .471 .923,5$ & 28 & 6.366 .511 .764 & 29 & 2.653 .349 .780 & 30 \\
\hline 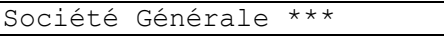 & 7.152 .378 .018 & 9 & $7.986 .033 .931,2$ & 15 & 6.614 .784 .747 & 26 & 3.431 .985 .749 & 15 \\
\hline 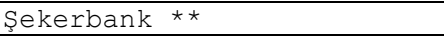 & 7.082 .115 .739 & 14 & $8.060 .254 .648,8$ & 12 & 6.739 .477 .753 & 13 & 3.416 .750 & 13 \\
\hline Tekstil Bankas & 7.096 .539 .969 & 13 & 7.934 .676 .884 & 17 & 6.688 .402 .750 & 16 & 3.431 .502 .743 & 16 \\
\hline $\begin{array}{l}\text { The Royal Bank of Scotland } \\
\text { Plc } \star \star \star\end{array}$ & 7.031 .888 .033 & 20 & $7.912 .185 .947,1$ & 22 & 6.619 .645 .754 & 23 & 3.377 .976 .748 & 22 \\
\hline Turkish Bank ** & & 21 & & 25 & & 25 & 3.387 .776 .741 & 21 \\
\hline Turkland Bank *** & 7.058 .588 .001 & 19 & $7.913 .118 .911,3$ & 21 & 3.559 .753 & 27 & 3.321 .133 .748 & 25 \\
\hline Türk Ekonomi Bankası ** & 7.862 .316 .264 & 5 & $8.460 .351 .181,4$ & 9 & 7.155 .646 .795 & 7 & 4.261 .080 .793 & 8 \\
\hline T.C. Ziraat Bankası * & - & 30 & - & 31 & - & 32 & - & 32 \\
\hline Türkiye Garanti Bankası ** & 7.986 .141 .646 & 3 & $9.897 .612 .516,9$ & 3 & 8.363 .458 .039 & 2 & 5.254 .480 .005 & 3 \\
\hline Türkiye Halk Bankası * & 5.835 .449 .903 & 29 & $6.051 .215 .707,8$ & 30 & 3.123 .542 .934 & 31 & 2.243 .979 .913 & 31 \\
\hline Türkiye İş Bankası ** & 6.865 .309 .989 & 24 & $9.547 .520 .933,0$ & 4 & 8.735 .945 .008 & 1 & 6.758 .176 .973 & 1 \\
\hline Türkiye Vakıflar Bankası * & 6.988 .692 .023 & 22 & $16.527 .953 .832,6$ & 1 & 7.131 .666 .936 & 8 & 5.068 .102 .922 & 4 \\
\hline Yapı ve Kredi Bankası ** & 8.715 .220 .794 & 1 & $10.355 .239 .713,3$ & 2 & 8.107 .740 .923 & 3 & 6.028 .731 .909 & 2 \\
\hline
\end{tabular}




\section{SONUÇ VE ÖNERILER (CONCLUSION AND RECOMMENDATIONS)}

OCRA temelli modelin bankacllık sektörüne uygulanması sonucunda dört yıl üzerinden yapılan değerlendirmede en etkin bankanın Yapı ve Kredi Bankası olduğu belirlenmiştir. İkinci sırayı Türkiye Garanti Bankası alırken üçüncü sırayı ise ING Bank'ın aldığı görülmektedir. Performansı en düşük bankanın ise Türkiye Cumhuriyeti Ziraat Bankası ile Türkiye Halk Bankası olduğu anlaşılmıştır. Çalışma sonucuna göre kamu sermayeli bankaların son sıralarda yer alması normal karşılanabilirken, Citibank'ın son sıralarda olması dikkat çekici bir durum olarak karşımıza çıkmaktadır. Diğer taraftan yabancı sermayeli, büyük sayılabilecek bankaların genellikle üst sıralara yakın yerlerde olduğu görülmektedir. Bu durum beklentimizin karşılık bulduğunu göstermektedir. Dikkat çekici bir başka durum olarak da kamu sermayeli bir banka olan Türkiye Vakıflar Bankasının 8. sırada yer almasıdır. Beklentilerin aksine Türkiye Vakıflar Bankasının pozisyonu önemsenmesi gereken bir durumdur. Akbank 2011 yılı dışında iyi bir performans göstermiştir. Finansbank, Türkiye Ekonomi Bankası performansı iyi sayılabilecek diğer bankalardır.

İleride yapılacak çalışmalarda uygulamaya koyduğumuz ORCA yöntemine dayanan model, VZA temeline dayalı başka bir modelle karşılaştırılabilir. Ayrıca son yıllarda çok kullanılan MOORA (MultiObjective ve Optimization on the basis of Ratio Analysis), VIKoR (VIse Kriterijumska Optimizacija I Kompromisno Resenje), Gri İlişki Analizi (GIA), TOPSIS gibi ÇÖKV yöntemleriyle de yapılan bu çalışma karşılaştırılabilir. Diğer taraftan girdi ve çıktı faktörleri değiştirilerek sonuçlar tekrar analiz edilebilir.

Model, zorlayıcı matematik bilgisi gerektirmeden Excel gibi paket programlar tarafından çözümlenebilmektedir. Bu açıdan sektör yöneticileri tarafından kolay uygulanabilir özelliğe sahiptir. Diğer taraftan model, bankacılık sektörü dışında da birçok farklı alanlarda girdi ve çıktı faktörleri değiştirilerek uygulanabilir.

\section{KAYNAKLAR (REFERENCES)}

1. Akkoç, S. and Vatansever, K., (2013). Fuzzy performance evaluation with AHP and TOPSIS methods: evidence for Turkish banking sector after the global financial crisis. Eurasian J. Bus. Econ. Vol:6, Nr:11, pp:53-74 (Available at: http: / / www . ejbe.org/EJBE2013Vol 06No11p053AKKOC-VATANSEVER.pdf) .

2. Ayadi, I. and Ellouze, A., (2013). Market Structure and Performance of Tunisian Banks. International Journal of Economics and Financial Issues, Vol:3, Nr:2, pp:345-354.

3. Bankalarımız 2011, Yayın No:284, G.M. Matbacılık ve Ticaret A.Ş. İstanbul, Mayıs 2012.

4. Bankalarımız 2012, Yayın No:294, G.M. Matbacılık ve Ticaret A.Ş. İstanbul, Mayıs 2013.

5. Bankalarımız 2013, Yayın No:304, G.M. Matbacılık ve Ticaret A.Ş. İstanbul, Mayıs 2014.

6. Bankalarımız 2014, Yayın No:311, G.M. Matbacılık ve Ticaret A.Ş. İstanbul, Mayıs 2015.

7. Bauer, P.W., Berger, A.N., Ferrier, G.D., and Humphrey, D.B., (1998). Consistency conditions for regulatory analysis of financial institutions: A comparison of frontier efficiency methods. Journal of Economic and Business, Vol:50, Nr:2, pp:85114 .

8. Bayyurt, N., (2013). Ownership Effect on Bank's Performance: Multi Criteria Decision Making Approaches on Foreign and 
Domestic Turkish Banks. Procedia-Social and Behavioral Sciences, 2013, 99: 919-928.

9. Beccalli, E., Casu, B., and Girardone, C., (2006). Efficiency and stock performance in european banking. Journal of Business Finance and Accounting, Vol:33, Br:1-2, pp:245-262.

10. Berger, A.N. and Humphrey, D.B., (1997). Efficiency of financial institutions: International survey and directions for future research. European Journal of Operational Research, Nr:98, pp: $175-212$.

11. Camanho, A.S. and Dyson, R.G., (2006). Data envelopment analysis and Malmquist indices for measuring performance. Journal of Productivity Analysis Nr:26, pp:35-49.

12. Che, Z.H.,Wang, H.S., and Chuang, C.L., (2010). A fuzzy AHP and DEA approach for marketing bank loan decisions for small and medium enterprises in Taiwan. Expert Syst. Appl., Vol:37, Nr:10, pp: 7189-7199.

13. Chen, Y.C., Chiu, Y.H., Huang, C.W., and Tu, C.H., (2013). The analysis of bank business performance and market risk - applying fuzzy DEA. Economic modeling, Nr:32, pp:225-232. http://dx. doi.org/10.1016/j.econmod.2013.02.008.

14. Chitan, G., (2012). Corporate governance and bank performance in the Romanian banking sector. Procedia Economics and Finance, $\mathrm{Nr}: 3, \mathrm{pp}: 549-554$.

15. Colwell, R.J. and Davis, E.P., (1992). Output and productivity in banking. The Scandinavian Journal of Economics, Nr:94, pp: $111-129$.

16. Daly, K. and Zhang, X., (2014). Comparative analysis of the performance of Chinese Owned Banks'in Hong Kong 20042010. Journal of Multinational Financial Management, NT:27, $\mathrm{pp}: 1-10$.

17. Demir, Y. and Astarcıoğlu, M., (2007). Determining bank performance via financial prediction: An application in ISE. Suleyman Demirel University. Journal of Business Administration and Economics Faculty, Vol:12, Nr:1, pp:273-292

18. Denizer, C., Dinc, M., and Tarımcllar, M., (2000). Measuring bank efficiency in the pre and post liberalization environment: Evidence from the Turkish banking system. World Bank Policy Research Working Paper, p:2476.

19. Doumpos, M. and Zopounidis, C., (2010), A multi criteria decision support system for bank rating. Decision Support Systems, Vol:50, Nr:1, pp:55-63.

20. Erdem, C. and Erdem, M.S., (2008). Turkish banking efficiency and its relation to stock performance. Applied Economics Letters Nr:15, pp:207-211.

21. García, F., Guijarro, F., and Moya, I., (2010). Ranking Spanish savings banks: A multi criteria approach. Mathematical and Computer Modelling Nr:52, pp:1058-1065.

22. Güven, S. and Persentili, E., (1997). A linear programming model for bank balance sheet management. Omega, Nr:25, pp:449-459.

23. Ho, C.T., (2006). Measuring bank operations performance: an approach based on grey relation analysis. Journal of the Operational Research Society Nr:57, pp:337-349.

24. Huang, T-H. and Wang, M-H., (2002). Comparison of economic efficiency estimation methods: Parametric and non-parametric techniques. The Manchester School, Vol:70, Nr:5, pp:682-709.

25. Ifeacho, C. and Ngalawa, H., (2014). Performance Of The South African Banking Sector Since 1994. Journal of Applied Business Research (JABR), Vol:30, Nr:4, pp:1183-1196. 
Özbek, A.

NWSA-Social Sciences, 3C0132, 10, (3), 120-134.

26. Ishizaka, A. and Nguyen, N.H., (2013). Calibrated fuzzy AHP for current bank selection. Expert Syst. Appl. Vol:40, Nr:9, pp:3775-3783. http://dx.doi.org/10.1016/j.eswa.2012.12.089.

27. Işık, I., Uysal, D., and Meleke, U., (2003). Post-entry performance of de novo banks in Turkey. In 10 th Annual conference of the ERF.

28. Jayanthi, S., Kocha, B., and Sinha, K.K., (1999).Competitive analysis of manufacturing plants: An application to the US processed food industry. European Journal of Operational Research, Vol:118, No:2, pp:217-234.

29. Kaya, Y.T., (2001). CAMELS analysis in Turkish banking sector. BRSA MSPD working report 6 .

30. Lin, T.-Y. and Chiu, S.-H., (2013). Using independent component analysis and network DEA to improve bank performance evaluation. Economic Modelling, Nr:32, pp:608-616.

31. Mahrooz, A., Maedeh, S., and Morteza, P., (2013). Performance evaluation of banks using fuzzy AHP and TOPSIS, case study: state-owned banks, particularly private and private banks in Iran. Caspain J. Appl. Sci. Res., Vol:2, Nr:3, pp:128.

32. Mandic, K., Delibasic, B., Knezevic, S., and Benkovic, S., (2014). Analysis of the financial parameters of Serbian banks through the application of the fuzzy AHP and TOPSIS methods. Economic Modelling, Nr:43, pp:30-37.

33. Mareschal, B. and Brans, J., (1991). BANKADVISER: an industrial evaluation system, European Journal of Operational Research Vol:54, Nr:3, pp:318-324.

34. Mareschal. B., and Mertens, D., (1992). BANKS: a multi criteria decision support system for financial evaluation in the international banking sector, Journal of Decision Systems, Vol:50, Nr:1, pp:175-189.

35. Özbek, A., (2015). Performance Analysis of Public Banks in Turkey, International Journal of Business Management and Economic Research(IJBMER), Vol:6, Nr:3, pp:178-186.

36. Parkan, C., (1999). Performance measurement in government services. Managing Service Quality, Vol:9, No:2, pp:121-135.

37. Parkan, C., (2002). Measuring the operational performance of a public transit company. International Journal of Operations \& Production Management, Vol:22, No:6, pp:693-720.

38. Parkan, C., (2005). Benchmarking operational performance: the case of two hotels. International Journal of Productivity and Performance Management, Vol:54. No:8, pp:679-696.

39. Parkan, C., Lam, K., and Hang, G., (1997). Operational competitiveness analysis on software development. Journal of the Operational Research Society, Vol:48, No:9, pp:892-905.

40. Parkan, C. and Wu, M., (1999). Measurement of the performance of an investment bank using the operational competitiveness rating procedure. Omega, Vol:27, Nr:2, pp:210-217.

41. Parkan, C. and Wu, M.-L., (1999a). Measuring the performance of operations of Hong Kong's manufacturing industries. European Journal of Operational Research, Vol:118, No:2, pp:235-258.

42. Parkan, C., Wu, M.-L., (1999b). Measurement of the performance of an investment bank using the operational competitiveness rating procedure. Omega, Vol:27, No:2, pp:201-217.

43. Parkan, C., (1994). Operational Competitiveness Ratings of Production Units. Managerial and Decision Economics, Vol:15, No:3, pp:201-221. 
Özbek, A.

44. Parkan, C., (2003). Measuring the effect of a new point of sale system on the performance of drugstore operations. Computers \& Operations Research, Vol:30, No:5, pp:729-744.

45. Parkan, C. and WU, M.-L., (2000). Comparison of three modern multi criteria decision-making tools. International Journal of Systems Science, 2000, Vol:31, Nr:4, pp:497-517.

46. Pasiouras, F., Liadaki, A., and Zopounidis, C., (2008). Bank efficiency and share performance: Evidence from Greece. Applied Financial Economics Vol:18, Nr:14, pp:1121-1130.

47. Peters, M.L. and Zelewski, S., (2010). Performance Measurement mithilfe des Operational Competitiveness Ratings (OCRA). WiSt, Wirtschaftswissenschaftliches Studium, Vol:39, Nr:5, p.224.

48. Portela, M.C.A.S. and Thanassoulis, E., (2007). Comparative efficiency analysis of Portuguese bank branches. European Journal of Operational Research Vol:177, Nr:2, pp:1275-1288.

49. Puhuong Ta, H. and Yin Har, K., (2000). A study of bank selection decisions in Singapore using the Analytical Hierarchy Process, International Journal of Bank Marketing, Vol:18, Nr:4, pp:170-180. http://dx.doi.org/10.1108/02652320010349058.

50. Sangmi, M. and Nazir, T., (2010). Analyzing financial performance of commercial banks in India:Application of CAMEL model. Pakistan Journal of Commerce and Social Sciences, Vol:4, $\mathrm{Nr}: 1, \mathrm{pp}: 40-55$.

51. Seçme, N.Y., Bayrakdaroğlu, A., and Kahraman, C., (2009). Fuzzy performance evaluation in Turkish banking sector using analytic hierarchy process and TOPSIS. Expert Syst. Appl., Vol:36, Nr:9, pp:11699-11709.

52. Spathis, C., Kosmidou, K., and Doumpos, M., (2002). Assessing profitability factors in the Greek banking system: a multi criteria approach. International Transactions in Operational Research, NR:9, pp:517-530.

53. Stankevičiené, J. and Mencaité, E., (2012). The evaluation of bank performance using a multi criteria decision making model: a case study on Lithuanian commercial banks. Technological and Economic Development of Economy, Vol:18, Nr:1, pp:189-205.

54. Thagunna, K.S. and Poudel, S., (2012). Measuring bank performance of Nepali banks: A Data envelopment analysis (DEA) perspective. International Journal of Economics and Financial Issues, Vol:3, Nr:1, pp:54-65.

55. Weifeng, X. and Huihuan, G., (2008). Using fuzzy analytic hierarchy process and balanced scorecard for commercial bank performance assessment. In Business and Information Management, IEEE, ISBIM'08. International Seminar on, Vol:1, pp:432-435. 